\title{
Composição, similaridade e estrutura do componente arbustivo- arbóreo de áreas de Caatinga
}

\author{
Alex da Silva BARBOSA루, Alberício Pereira de ANDRADE², Leonardo Pessoa FÉLIX ${ }^{3}$, \\ Ítalo de Souza AQUINO1 ${ }^{1}$, João Henrique Constantino Sales SILVA4*
}

\author{
${ }^{1}$ Centro de Ciências Humanas, Sociais e Agrárias, Universidade Federal da Paraíba, Bananeiras, PB, Brasil. \\ (ORCID: 0000-0002-7343-6134; 000-0002-7948-8760) \\ 2Universidade Federal Rural de Pernambuco, Garanhuns, PE, Brasil. (ORCID: 0000-0002-1223-394X) \\ 3 Programa de Pós-Graduação em Agronomia, Universidade Federal da Paraíba, Areia, PB, Brasil. (ORCID: 0000-0001-9202-9828) \\ ${ }^{4}$ Programa de Pós-Graduação em Ciências Agrárias (Agroecologia), Universidade Federal da Paraíba, Bananeiras, PB, Brasil. \\ *E-mail: joaohenriqueconst@gmail.com (ORCID: 0000-0001-6218-5096)
}

Recebido em 09/12/2019; Aceito em 04/04/2020; Publicado em 24/04/2020.

\begin{abstract}
RESUMO: Os fragmentos de caatinga constituem áreas que necessitam de estudos buscando subsidiar ações de manejo e conservação desses habitats. O objetivo deste estudo foi analisar a florística e a estrutura de três áreas de caatinga localizadas nos municípios de Campina Grande, Boa Vista e Monteiro - PB. Em cada área foram plotadas 100 parcelas de $100 \mathrm{~m}^{2}$, sendo amostrados e etiquetados todos os indivíduos de porte arbustivoarbóreo com circunferência ao nível do solo $\geq 9 \mathrm{~cm}$ e altura total $\geq 1 \mathrm{~m}$. Todo o material vegetal foi coletado e enviado para identificação no Centro de Ciências Agrárias (CCA/UFPB) "Herbário Jayme Coelho de Moraes" (EAN), por meio de comparação com exsicatas e literatura especializada. O processamento dos dados para obtenção dos descritores fitossociológicos e similaridade florística foi realizado no programa Mata Nativa $2^{\circledR}$. As famílias mais representativas nas áreas foram Euphorbiaceae, Fabaceae e Cactaceae, sendo as espécies mais representativas Croton blanchetianus Baill., Poincianella pyramidalis (Tul.) L.P. Queiroz., Mimosa ophthalmocentra Mart. ex Benth. e Tacinga palmadora (Britton \& Rose) N. P. Taylor \& Stuppy. A caatinga de Monteiro apresentou maior diversidade florística $\left(H^{\prime}=2,3\right.$ nats. ind. $\left.{ }^{-1}\right)$ e melhor estado de conservação do patrimônio genético autóctone em relação às áreas de Campina Grande $\left(H^{\prime}=1,98\right.$ nats. ind. $\left.{ }^{-1}\right)$ e Boa Vista, PB $\left(H^{\prime}=1,78\right.$ nats. ind..$\left.^{-1}\right)$. As áreas de caatinga apresentam algum grau de intervenção antrópica. Todas as áreas são similares floristicamente.

Palavras-chave: florística; fitossociologia; vegetação nativa.
\end{abstract}

\section{Composition, structure and similarity of component shrub-arboreal of Caatinga areas}

\begin{abstract}
The caatinga's fragments areas still need of studies to support management actions and conservation of their habitats. The aim of this study was to analyze the floristic and structure of three caatinga's areas in the counties of Campina Grande, Boa Vista and Monteiro - PB. In each area were plotted 100 plots with $100 \mathrm{~m}^{2}$, being sampled and tagged all individuals of shrub-tree with a $\geq 9 \mathrm{~cm}$ circumference at ground level and $\geq 1 \mathrm{~m}$ height. All the plant material was collected and sent for identification at the Center for Agricultural Sciences (UFPB/CCA) "Herbarium Jaime Coelho de Moraes" (EAN), by comparison with herbarium specimens and literature. Data processing to obtain the phytosociological descriptors and floristic similarity was performed by using the Mata Nativa $2^{\circledR}$ program. The most representative families found in these three locations were Euphorbiaceae, Fabaceae and Cactaceae, being the most representative species identified were: Croton blanchetianus Baill., Poincianella pyramidalis (Tul.) L.P. Queiroz., Mimosa ophthalmocentra Mart. ex Benth. and Tacinga palmadora (Britton \& Rose) N.P. Taylor \& Stuppy. The caatinga of Monteiro showed greater floristic diversity $\left(H^{\prime}=2.3\right.$ nats.ind $\left.{ }^{-1}\right)$ and better state of conservation of genetic resources in relation to autochthonous areas when compared to Campina Grande $\left(H^{\prime}=1.98\right.$ nats.ind.$\left.^{-1}\right)$ and Boa Vista, $\mathrm{PB}\left(H^{\prime}=1.78\right.$ nats.ind. $\left.{ }^{-1}\right)$. The caatinga areas have some degree of human intervention. All areas are similar floristically.
\end{abstract}

Keywords: floristic; phytosociology; native vegetation.

\section{INTRODUÇÃO}

A Caatinga é considerada pelo Ministério do Meio Ambiente como um dos grandes biomas brasileiros, abrangendo $734 \mathrm{mil} \mathrm{km}^{2}$ (SILVA et al., 2004), detentora de uma diversidade florística alta para um bioma com uma restrição forte ao crescimento vegetal, consequência da variabilidade da precipitação pluvial. Áreas de caatingas típicas, em geral, têm menos de 50 espécies de arbustivas e arbóreas e igual número de herbáceas por hectare (GARIGLIO et al., 2010). A Caatinga no seu sentido mais restrito teria 1.512 espécies com no mínimo 318 endêmicas (GARIGLIO et al., 2010). 
De acordo com Giulietti (2006), as principais famílias da caatinga, considerando números de espécies, são: Leguminosae (278 espécies), Convolvulaceae (103 espécies), Euphorbiaceae (73 espécies), Malpighiaceae (71 espécies), Poaceae (66 espécies) e Cactaceae (57 espécies). Rodal et al. (1992) destaca que apesar da existência de trabalhos relacionados com a caatinga são necessários estudos com sua vegetação, havendo necessidade de se realizar levantamentos das espécies, determinar seus padrões de distribuição geográfica, abundância e relação com os fatores ambientais. Esses estudos estabelecerão com base em dados quantitativos, os diferentes tipos de caatinga e suas conexões florísticas.

Segundo Marangon et al. (2003), o conhecimento e o entendimento da complexa dinâmica que envolve as florestas iniciam-se pelo levantamento da florística. A identidade das espécies e o seu comportamento em comunidades vegetais são o começo de todo processo para compreensão deste ecossistema. Além disso, o levantamento florístico pode ser considerado um importante instrumento para a avaliação dos fragmentos arbóreo-arbustivos existentes em uma determinada área, bem como para subsidiar tomadas de decisão quanto aos eventuais cortes de árvores para utilização da biomassa vegetal para a retirada de lenha e madeira, dentre outros aspectos para fins de interesse socioeconômicos (ARAÚJO et al., 2010).

Considerando a importância de conhecimentos que definam a estrutura e o funcionamento dos ecossistemas da caatinga, torna-se imprescindível a necessidade de estudos sobre os seus aspectos florísticos e fitossociológicos, para o conhecimento pormenorizado de grupos taxonômicos, a relação entre esses e com o ambiente em que vivem. $\mathrm{O}$ objetivo deste estudo foi analisar a composição florística, estrutura, padrão de distribuição de espécies autóctones e similaridade de fragmentos de caatinga localizados nos municípios de Campina Grande, Boa Vista e Monteiro - PB.

\section{MATERIAL E MÉTODOS}

\section{1. Áreas de estudo}

A primeira área está localizada no Instituto Nacional do Semiárido (INSA/MCT), no município de Campina Grande - PB, situado nas coordenadas $7^{\circ} 16^{\prime} 35,97^{\prime \prime S}$ e $35^{\circ} 57^{\prime} 55,72^{\prime \prime} \mathrm{O}$. A vegetação predominante é formada pela floresta subcaducifólia e caducifólia, a qual se modifica drasticamente ao longo das estações seca e chuvosa. O clima de acordo com a classificação de Köppen é do tipo 'As', quente e úmido, com chuvas de outono-inverno (CPRM, 2005a). A média da precipitação pluvial aproximada é de $800 \mathrm{~mm}$. Segundo informações dos moradores da região a área foi utilizada há 25 anos para os cultivos de algodão e agave; na época era de propriedade particular onde muitos trabalhavam como assalariados.

A segunda área utilizada é de propriedade particular, denominada de Sítio Caldeirão localizada no Município de Boa Vista - PB, situada nas seguintes coordenadas $7^{\circ} 10^{\prime} 21,09^{\prime \prime S}$ e $36^{\circ} 9^{\prime} 55,03$ "O. A área foi adquirida por meio de herança familiar e sempre foi mantida conservada, com finalidade de pastoreio controlado em época de escassez de alimentos para os animais. A vegetação da área é formada por espécies subcaducifólia e caducifólia, onde há pelo menos 30 anos não foi utilizada para agricultura. O clima predominante, segundo a classificação de Köppen é BSh, quente seco, com precipitações locais que não ultrapassam 500mm (GOVERNO DO ESTADO DA PARAÍBA, 2006).

A terceira área é de propriedade particular, denominada Sítio Mocó de Baixo, situada no município de Monteiro - PB, nas coordenadas $7^{\circ} 10^{\prime} 23,1^{\prime \prime}$ e e $36^{\circ} 09^{\prime} 53,4^{\prime \prime} \mathrm{O}$. O clima é BSh, semiárido quente, com chuvas de verão, segundo a classificação de Köppen, com precipitação média pluvial anual de 431,8 $\mathrm{mm}$. As médias de temperatura dificilmente são inferiores a $24^{\circ} \mathrm{C}$. A vegetação que recobre a região é a caatinga hiperxerófila (vegetação caducifólia espinhosa), com trechos de floresta caducifólia (CPRM, 2005b).

\subsection{Coleta de dados e levantamento da vegetação}

O método utilizado para coleta dos dados foi o de parcelas contíguas (MULLER-DOMBOIS; ELLEMBERG, 1974; RODAL et al., 1992) em área de um hectare. Esse método vem sendo utilizado para o estudo da caatinga, e permite inferências mais apropriadas para determinados aspectos da vegetação em estudo. As três áreas foram divididas em 100 parcelas de $10 \mathrm{~m}$ x $10 \mathrm{~m}$, totalizando uma área amostral de $30.000 \mathrm{~m}^{2}$ (três hectares).

O levantamento florístico foi realizado por meio de caminhamento nas parcelas, onde foram coletadas partes botânicas das plantas amostradas com presença aparente de botões florais e flores abertas. Os espécimes coletados em campo foram herborizados e, em seguida, confeccionadas as exsicatas. O material coletado passou pelo processo de secagem, onde os ramos foram intercalados entre folhas de jornais na prensa e armazenados em estufa de circulação de ar a $65^{\circ} \mathrm{C}$ durante $24 \mathrm{~h}$. As exsicatas foram enviadas para o Centro de Ciências Agrárias (CCA) "Herbário Jayme Coelho de Moraes" (EAN/UFPB), para identificação dos indivíduos por meio de comparação com materiais que fazem parte da coleção do herbário, como também, foram feitas comparações com a literatura especializada. A sinonímia e a grafia dos táxons foram atualizadas mediante consulta ao índice de espécies do banco de dados Tropicos ${ }^{\circledR}$ do Missouri Botanical Garden (Disponível em: $<$ http://www.tropicos.org/>). A lista florística foi organizada de acordo com o sistema Angiosperm Phylogeny Group IV (APG IV) (THE ANGIOSPERM PHYLOGENY GROUP et al., 2016).

Para a análise da heterogeneidade florística da área foi utilizado o índice de diversidade específica de Shannon $(H)$ e o índice de equabilidade $(J)$, de acordo com Magurran (1988) e Pielou (1975). A análise de similaridade florística foi realizada de acordo com o índice qualitativo (presença/ausência) de Jaccard e a agregação de acordo com índice de McGuinnes. A determinação do índice de agregação foi realizada através da equação:

$$
\mathrm{IGA}=\frac{\mathrm{D}}{\mathrm{d}}
$$

em que: $\mathrm{D}=$ número total de indivíduos da espécie/número total de parcelas alocadas; $\mathrm{d}=-\ln (1-\mathrm{FA} / 100)(\ln =\operatorname{logaritmo}$ neperiano e FA = Frequência Absoluta)

Em cada parcela foram amostrados e etiquetados com placas de alumínio todos os indivíduos de porte arbustivoarbóreo. Foi mensurada a altura do caule principal considerando a copa com o auxílio de uma vara tubular graduada de todos os indivíduos com altura $\geq$ a $1 \mathrm{~m} \mathrm{e}$ 
circunferência ao nível da base $\geq$ a $9 \mathrm{~cm}$, com auxílio de uma fita métrica. Esse critério foi utilizado, pois na literatura é frequente a utilização dessa metodologia, e, por conseguinte permitir a comparação com outras fitofisionomias da caatinga.

Os descritores estruturais analisados foram: densidade absoluta e relativa (DA e DR), frequência absoluta e relativa (FA e FR), dominância absoluta e relativa (DoA e DoR), valor de importância (VI) e o valor de cobertura (VC) para cada espécie amostrada, obtidos com auxílio do programa Mata Nativa $2^{\circledR}$ (CIENTEC, 2006).

\section{RESULTADOS}

\subsection{Florística}

A vegetação arbustivo-arbórea das três áreas amostradas foi representada por 64 espécies (Tabela 1). M. urundeuva, $S$. tuberosa, A. pyrifolium, C. leptophloeos, C. jamacaru, M. dicotoma, M. glaziovii, P. pyramidalis, A. colubrina var cebil, $M$. ophthalmocentra e $A$. quercifolius foram as espécies de ampla ocorrência nas três áreas.

$\mathrm{Na}$ caatinga do INSA/MCT foram amostradas 31 espécies, distribuídas em 14 famílias e 26 gêneros. As famílias mais representativas foram Fabaceae $(\mathrm{N}=11)$, Euphorbiaceae $(\mathrm{N}=4)$, Anacardiaceae $(\mathrm{N}=2)$, Cactaceae $(\mathrm{N}=2)$, Capparaceae $(\mathrm{N}=2)$ e Malvaceae $(\mathrm{N}=2)$. Essas famílias representaram $76 \%$, sendo que as demais oito famílias representaram $24 \%$ da área (Tabela 1).

No Sítio Caldeirão foram amostradas 32 espécies, distribuídas em 14 famílias e 28 gêneros. As famílias mais representativas foram Fabaceae $(\mathrm{N}=9)$, Cactaceae $(\mathrm{N}=6)$, Anacardiaceae $(\mathrm{N}=3)$, Euphorbiaceae $(\mathrm{N}=3)$ e Malvaceae $(\mathrm{N}=2)$, representando $73 \%$ da área, sendo que as demais famílias representaram $27 \%$ com uma espécie para cada família (Tabela 1).

No Sítio Mocó de Baixo foram amostradas 37 espécies, distribuídas em 15 famílias e 34 gêneros. As famílias mais representativas foram Fabaceae $(\mathrm{N}=10)$, Euphorbiaceae $(\mathrm{N}=5)$, Anacardiaceae $(\mathrm{N}=3)$, Cactaceae $(\mathrm{N}=3)$, Annonaceae $(\mathrm{N}=2)$ e Malvaceae $(\mathrm{N}=2)$, correspondendo a $67,5 \%$ da área. O restante esteve compreendido com $32,5 \%$ de famílias com um representante cada uma (Tabela 1$)$.

A análise realizada com base no índice de similaridade demonstrou coeficiente de 0,97 entre a área de Campina Grande e Boa Vista, 0,71 entre as áreas de Boa Vista e Monteiro e 0,69 entre as áreas de Campina Grande e Monteiro.

\subsection{Estrutura da vegetação}

Nas parcelas inventariadas na área do INSA/MCT foram amostrados 3.526 indivíduos distribuídos 31 espécies, distribuídas em 14 famílias e 26 gêneros. De modo geral, observou-se que dentre as 14 famílias presentes na área, Euphorbiaceae apresentou o maior número de indivíduos $(\mathrm{N}=1.506)$ seguida por Fabaceae $(\mathrm{N}=1.385)$ e Sapindaceae $(\mathrm{N}=327)$. As Euphorbiaceae foram representadas principalmente por indivíduos C. blanchetianus, M. dichotoma, C. rhamnifolioides e J. bahiana.

Os descritores estruturais demonstraram que $C$. blanchetianus apresentou maior densidade absoluta $\left(\mathrm{DA}=1.431\right.$ ind.ha $\left.\mathrm{a}^{-1}\right)$, frequência absoluta de $98 \%$ e, por conseguinte alto valor de importância (Tabela 2). Outra espécie representativa foi a $M$. ophthalmocentra com densidade absoluta de 730 ind. ha ${ }^{-1}$, frequência absoluta de $99 \%$ e valor de importância de $14,74 \%$, seguida de $P$. pyramidalis com densidade absoluta de 230 ind.ha ${ }^{-1}$, frequência absoluta $72 \%$ e valor de importância 9,81\%. Observou-se que as espécies C. blanchetianus, A. quercifolius, Mimosa sp.1, B. forficata, apresentaram padrão de distribuição agregada, de acordo com o índice de McGuinnes. Os espécimes de $M$. ophthalmocentra, P. pyramidalis, A. pyrifolium, P. pachiycladus, C. jamacaru, L. camara, M. dichotoma, M. tenuiflora, Acacia sp. $C$. leptophloeos, C. flexuosa, J. bahiana, Guapira sp., Eugenia sp., C. bexandra e Mimosa sp.3 apresentaram tendência ao agrupamento. As espécies com maior valor de importância foram C. blanchetianus (27,09\%), M. ophthalmocentra (14,74\%) e $P$. pyramidalis $(9,81 \%)$ (Tabela 2$)$.

Com base nessa estrutura pode-se inferir que a comunidade em estudo está em processo de estabelecimento, possuindo características de resiliência aos fatores antropogênicos. A diversidade encontrada em Campina Grande foi 1,98 nats.ind. ${ }^{-1}$ e a equabilidade de 0,58.

No Sítio Caldeirão, em Boa Vista, foram amostrados um total 3.600 espécimes distribuídos em 32 espécies, 14 famílias e 28 gêneros. A maior parte dos indivíduos pertencia às famílias Cactaceae $(\mathrm{N}=2.099)$, Fabaceae $(\mathrm{N}=862)$ e Combretaceae $(\mathrm{N}=336)$. As famílias com menores representantes taxonômicos foram Celastraceae $(\mathrm{N}=3)$ e Verbenaceae $(\mathrm{N}=3)$.

Os descritores estruturais demonstraram alta densidade absoluta de T. palmadora (DA=1.883 ind. $\mathrm{ha}^{-1}$ ) seguida de $P$. pyramidalis (DA=502 ind. ha ${ }^{1}$ ) e $P$. pachycladus (DA=171 ind. $\left.\mathrm{ha}^{-1}\right)$. As espécies que apresentaram menor densidade absoluta foram S. tuberosa, $M$. zehntneri e C. jamcaru (DA= 1 ind. ha $\left.{ }^{-1}\right)($ Tabela 3).

A análise de agregação demonstrou que as espécies $T$. palmadora, A. cearensis, S. brasiliensis, $M$. urundewva, $M$. rigida, $S$. tuberosa, H. eichleri, C. ferrea var. ferrea, $M$. zehntneri e C. jamacaru apresentaram padrão de distribuição uniforme de acordo com o índice de McGuinnes.

As espécies de maior valor de importância na área estudada foram T. palmadora (27,68\%), P. pyramidalis (20,1\%) e $P$. pachycladus $(9,94 \%)$. Os espécimes que apresentaram menor valor de importância na área foram C. jamacaru $(0,07 \%)$, L. camara $(0,08 \%)$ e M. zehtneri $(0,08 \%)$ (Tabela 3). $\mathrm{O}$ índice de diversidade encontrado para a área de Boa Vista foi de 1,78 nats.ind. ${ }^{-1}$ e a equabilidade de Pielou foi de 0,51.

No Sítio Mocó de Baixo, em Monteiro, foram amostrados 3.501 indivíduos, 34 espécies distribuídas em 15 famílias e 28 gêneros. A família com maior número de indivíduos foi a Euphorbiaceae $(\mathrm{N}=1.972)$, seguida de Fabaceae $(\mathrm{N}=1.081)$ e Malphighiaceae $(\mathrm{N}=183)$. As famílias com menor número de espécies foram Apocynaceae $(\mathrm{N}=4)$, Rhamnaceae $(\mathrm{N}=3)$ e Boraginaceae $(\mathrm{N}=2)$.

Os indivíduos com maior densidade absoluta na área estudada foram C. rhamnifolioides $(\mathrm{DA}=1086)$, B. cheilanta $(\mathrm{DA}=395)$ e $C$. blanchetianus ( $\mathrm{DA}=637)$ (Tabela 4). As espécies com padrão agregado foram C. rhamnifolioides, $C$. blanchetianus, B. cheilantha e $\mathrm{R}$. laurifolia. C. leptophloeos, $C$. foliolosum, S. brasiliensis, H. adscendens, Rollinia sp., S. macranthera, Z. joazeiro, T. cearensis, C. leucocephala, sp. 4, P. piaubyensis sp. 3, S. lanceolatum, sp. 5, e $S$. tuberosa apresentaram padrão de distribuição uniforme, enquanto todas as demais espécies amostradas apresentaram tendência à agregação. As espécies que apresentaram maiores valores de importância foram $P$. pyramidalis $(15,71 \%), \quad$ C. blanchetianus $(11,82 \%)$ e $M$. ophthalmocentra $(10,41 \%)$. 


\section{Barbosa et al.}

O índice de diversidade encontrado para a área de Monteiro foi de 2,3 nats.ind. ${ }^{-1}$ e a equabilidade de Pielou foi de 0,63. A caatinga de Monteiro apresentou maior diversidade florística seguida de Boa Vista e Campina Grande. As espécies de ocorrência estrita em Monteiro foram: $H$. adscendens, P. piaubyensis, Rollinia sp., R. laurifolia, L. auriculata, S. macranthera, sp. 1, C. glaziovii, H. ovata, Guapira sp. 2, e $H$. apiculata. P. gounellei, T. inamoena, T. palmadora, $C$. duarteanum, A. cearensis, C. parviflorum, e M. Zehntneri foram amostradas apenas em Boa Vista, sendo que C. yco, $M$. paraibana, Mimosa sp. 1, Mimosa sp. 2, Mimosa sp. 3, e C. hexandra amostradas apenas em Campina Grande.

Tabela 1. Famílias e espécies registradas nas áreas de caatinga nos municípios de Campina Grande (Cg), Boa Vista (Bv) e Monteiro (Mt) PB (0 - Ausência; 1 - Presença).

Table 1. Families and species recorded in the areas of natural vegetation in the cities of Campina Grande (Cg), Boa Vista (Bv) and Miller (Mt) - PB (0 - Absence; 1 - Presence).

\begin{tabular}{|c|c|c|c|c|}
\hline \multirow{2}{*}{ Divisão/Família/Espécie } & \multirow{2}{*}{ Nome Vernacular } & \multicolumn{3}{|c|}{ Áreas } \\
\hline & & $\mathrm{Cg}$ & $\mathrm{Bv}$ & Mt \\
\hline \multicolumn{5}{|l|}{ ANGIOSPERMAE } \\
\hline \multicolumn{5}{|l|}{ ANACARDIACEAE } \\
\hline Myracrodruon urundewva Fr. All. & Aroeira & 1 & 1 & 1 \\
\hline Schinopsis brasiliensis Engl. & Baraúna & 0 & 1 & 1 \\
\hline Spondias tuberosa Arr. Câm. & Umbuzeiro & 1 & 1 & 1 \\
\hline \multicolumn{5}{|l|}{ ANNONACEAE } \\
\hline Rollinia laurifolia Schldl. & Pinha-brava & 0 & 0 & 1 \\
\hline Rollinia sp. & Bananinha & 0 & 0 & 1 \\
\hline \multicolumn{5}{|l|}{ APOCYNACEAE } \\
\hline Aspidosperma pyrifolium Mart. & Pereiro & 1 & 1 & 1 \\
\hline \multicolumn{5}{|l|}{ BORAGINACEAE } \\
\hline Cordia lencocephala Moric. & Moleque-duro & 0 & 0 & 1 \\
\hline \multicolumn{5}{|l|}{ BURSERACEAE } \\
\hline Commiphora leptophloeos (Mart.) J.B. Gillett & Imburana & 1 & 1 & 1 \\
\hline \multicolumn{5}{|l|}{ CACTACEAE-CACTOIDEAE } \\
\hline Cereus jamacaru DC. & Mandacaru & 1 & 1 & 1 \\
\hline Harrisia adscendens (Gürke) Britton \& Rose & Rabo-de-raposa & 0 & 0 & 1 \\
\hline Melocactus zehntneri (Britton \& Rose) Luetzelb. & Coroa-de-frade & 0 & 1 & 0 \\
\hline Pilosocereus gounellei A. Weber ex K. Schum. & Xiquexique & 0 & 1 & 0 \\
\hline P. pacbycladus Ritter. & Facheiro & 1 & 1 & 0 \\
\hline P. piaubyensis (Gürke) Byles \& G. D. Rowley & Facheiro-dourado & 0 & 0 & 1 \\
\hline \multicolumn{5}{|l|}{ CACTACEAE-OPUNTIOIDEAE } \\
\hline Tacinga inamoena (K. Schum) N. P. Taylor \& Stuppy & Quipá & 0 & 1 & 0 \\
\hline T. palmadora (Britton et Rose) N. P. Taylor \& Stuppy & Palmatória & 0 & 1 & 0 \\
\hline \multicolumn{5}{|l|}{ CAPPARACEAE } \\
\hline Capparis cynophallophora $\mathrm{L}$. & Feijão-bravo & 0 & 0 & 1 \\
\hline C. flexuosa L. & Feijão-bravo & 1 & 0 & 0 \\
\hline C. yco (Mart.) Eichler & Icó & 1 & 0 & 0 \\
\hline \multicolumn{5}{|l|}{ CELASTRACEAE } \\
\hline Maytenus rigida Mart. & Bom nome & 0 & 1 & 0 \\
\hline \multicolumn{5}{|l|}{ COMBRETACEAE } \\
\hline Combretum duarteanum Cambess. & Cipaúba & 0 & 1 & 0 \\
\hline \multicolumn{5}{|l|}{ EUPHORBIACEAE } \\
\hline Croton blanchetianus Bail. & Marmeleiro & 1 & 0 & 1 \\
\hline C. rhamnifolioides Pax \& Hoffm. & Catinga-branca & 1 & 0 & 1 \\
\hline Jatropha bahiana Ule & Pinhão-bravo & 1 & 0 & 0 \\
\hline J. mollissima (Pohl) Baill. & Pinhão-bravo & 0 & 1 & 1 \\
\hline Manibot dichotoma Ule & Maniçoba & 0 & 0 & 1 \\
\hline M. gląiovii Muell. Arg. & Maniçoba & 1 & 1 & 0 \\
\hline Sapium lanceolatum Huber & Burra-leiteira & 0 & 1 & 1 \\
\hline \multicolumn{5}{|l|}{ FABACEAE-CAESALPINIOIDEAE } \\
\hline Caesalpinea ferrea Mart. ex Tul. var ferrea & Jucá & 1 & 1 & 0 \\
\hline Cbloroleucon foliolosum (Benth) G. P. Lewis & Jurema-açú & 0 & 0 & 1 \\
\hline \multicolumn{5}{|l|}{ FABACEAE-PAPILIONOIDEAE } \\
\hline Luetzelburgia auriculata (Allemão) Ducke & Pau-mocó & 0 & 0 & 1 \\
\hline Poincianella pyramidalis (Tul.) L. P. de Queiroz & Catingueira & 1 & 1 & 1 \\
\hline Senna macranthera (DC. ex Collad.) H. S. Irwin \& Barneby & Canudo-de-São-João & 0 & 0 & 1 \\
\hline \multicolumn{5}{|l|}{ FABACEAE-CERCIDEAE } \\
\hline Baubinia cheilantha (Bong.) Setend. & Mororó & 0 & 0 & 1 \\
\hline B. forficata Link & Mororó & 1 & 1 & 0 \\
\hline
\end{tabular}


Tabela 1. Famílias e espécies registradas nas áreas de caatinga nos municípios de... (CONTINUAÇÃO).

Table 1. Families and species recorded in the areas of natural vegetation in the cities... (CONTINUATION)).

\begin{tabular}{|c|c|c|c|c|}
\hline \multirow{2}{*}{ Divisão/Família/Espécie } & \multirow{2}{*}{ Nome Vernacular } & \multicolumn{3}{|c|}{ Áreas } \\
\hline & & $\mathrm{Cg}$ & $\mathrm{Bv}$ & Mt \\
\hline \multicolumn{5}{|l|}{ FABACEAE-FABOIDEAE } \\
\hline Amburana cearensis (Allemão) A. C. Smith & Cumarú & 0 & 1 & 0 \\
\hline Torresea cearensis (Fr. All.) A. C. Smith & Imburana-de-cheiro & 0 & 0 & 1 \\
\hline \multicolumn{5}{|l|}{ FABACEAE-MIMOSOIDEAE } \\
\hline Acacia sp. & Jurema-papagaio & 1 & 1 & 0 \\
\hline Anadenanthera colubrina (Vell.) Brenan var cebil (Griseb.) Altshul & Angico-de-caroço & 1 & 1 & 1 \\
\hline Mimosa adenophyla Taub. & Amorosa-branca & 0 & 1 & 0 \\
\hline M. opbthalmocentra Mart. ex Benth. & Jurema-de-imbira & 1 & 1 & 1 \\
\hline M. tenuiflora (Willd.) Poir. & Jurema-preta & 1 & 1 & 1 \\
\hline M. paraibana Barneby & Camuzé & 1 & 0 & 0 \\
\hline Mimosa sp. 1 & Jurema-unha-de-gato & 1 & 0 & 0 \\
\hline Mimosa sp. 2 & Jurema-catinguenta & 1 & 0 & 0 \\
\hline Mimosa sp. 3 & Jurema-de-feitiçeiro & 1 & 0 & 0 \\
\hline Parapiptadenia zehntneri (Harms) M.P. Lima \& Lima & Angico-manjola & 0 & 0 & 1 \\
\hline \multicolumn{5}{|l|}{ MALPIGHIACEAE } \\
\hline sp. 1 & Catinga-branca & 0 & 0 & 1 \\
\hline \multicolumn{5}{|l|}{ MALVACEAE } \\
\hline Ceiba glaziovii (Kuntze) K. Schum. & Barriguda & 0 & 0 & 1 \\
\hline Pseudobombax marginatum (St.-Hilll., Juss.\& Camb.) A. Robyns & Imbiratanha & 1 & 1 & 0 \\
\hline Helicteres eicbleri $\mathrm{K}$. Schum & Maria-Preta & 1 & 1 & 0 \\
\hline H. ovata Lam. & Velame & 0 & 0 & 1 \\
\hline \multicolumn{5}{|l|}{ MYRTACEAE } \\
\hline Eugenia sp. & Uvaia & 1 & 1 & 0 \\
\hline \multicolumn{5}{|l|}{ NYCTAGINACEAE } \\
\hline Guapira sp. 1 & João-Mole & 1 & 1 & 0 \\
\hline Guapira sp. 2 & Pau-Piranha & 0 & 0 & 1 \\
\hline \multicolumn{5}{|l|}{ RHAMNACEAE } \\
\hline Ziziphus joazeiro Mart. & Juazeiro & 1 & 0 & 1 \\
\hline \multicolumn{5}{|l|}{ RUTACEAE } \\
\hline Helietta apiculata Benth. & Canela-de-veado & 0 & 0 & 1 \\
\hline \multicolumn{5}{|l|}{ RUBIACEAE } \\
\hline Coutarea hexandra (Jacq.) K. Schum. & Quina-quina & 1 & 0 & 0 \\
\hline \multicolumn{5}{|l|}{ SAPINDACEAE } \\
\hline Allophylus quercifolius Radlk. & Murta & 1 & 1 & 1 \\
\hline \multicolumn{5}{|l|}{ SOLANACEAE } \\
\hline Capsicum parviflorum Sendtn. & Pimenta-de-macaco & 0 & 1 & 0 \\
\hline \multicolumn{5}{|l|}{ VERBENACEAE } \\
\hline Lantana camara $\mathrm{L}$. & Chumbinho & 1 & 1 & 0 \\
\hline \multicolumn{5}{|l|}{ INDETERMINADAS } \\
\hline sp. 3 & & 0 & 0 & 1 \\
\hline sp. 4 & & 0 & 0 & 1 \\
\hline sp. 5 & & 0 & 0 & 1 \\
\hline
\end{tabular}

\section{DISCUSSÃO}

As caatingas de Campina Grande e Monteiro apresentaram o menor índice de similaridade, sendo essas áreas as mais distantes, com características físicas de solos e histórico de uso das áreas contrastantes. Barbosa (2011) observou que a influência das características de solo sobre a pequena variação da vegetação está associada mais as características físicas de solo do que as químicas, uma vez que o solo muda de uma área para outra, no entanto os atributos físicos tem pouca variação entre as três áreas estudadas. Entretanto, por estarem dentro de um mesmo domínio vegetacional as áreas são similares floristicamente. Para Mueller-Dombois e Ellemberg (1974), áreas com índice de Jaccard superior a 0,25 são consideradas similares floristicamente.

As espécies Croton sp. e Mimosa sp. apresentaram maior valor de importância na área do INSA/MCT, sendo característica de áreas em estágio sucessional. A forte intervenção antrópica há duas décadas na área resultou na redução da riqueza florística do fragmento, corroborando para demonstrar a resiliência da caatinga, essas espécies atuam como colonizadoras primárias e secundárias, respectivamente, preparando o ambiente para as espécies típicas de comunidades estabelecidas ou clímax. A diversidade florística, em Campina Grande, encontrou-se próxima daquela encontrada por Pessoa (2008), quando trabalhou numa caatinga em estágio de sucessão ecológica.

Souza et al. (2019) ao caracterizar a variabilidade de uma vegetação nativa de Caatinga no Cariri Paraibano, constataram que as espécies mais predominantes foram: $P$. pyramidalis, A. pyrifolium, P. gounellei, $C$. sonderianus e J. molíssima para todas as áreas em estudo. De acordo com esses autores, duas áreas apresentaram 46\% de táxons da família Fabaceae e a diversidade e riqueza de famílias e espécies encontradas nas áreas de estudo foram baixas com presença dominante da espécie P. pyramidalis. Silva et al. (2015) também constataram que a família Fabaceae foi a mais representativa, seguida da Annonaceae, ao avaliarem o status da flora de um remanescente de mata ciliar em uma área de transição cerrado-caatinga no estado do Piauí. Ainda de acordo com esses autores, este fragmento apresenta um grande número de indivíduos e espécies, visto que a densidade foi de 3.445 ind.ha ${ }^{-1}$. 
Tabela 2. Espécies amostradas na área de vegetação natural do INSA/MCT, Campina Grande - PB, com os respectivos parâmetros fitossociológicos em ordem decrescente de valor de importância.

Table 2. Species sampled in the area of natural vegetation of INSA/MCT, Campina Grande - PB, with their phytosociological parameters and their value in descending order of importance.

\begin{tabular}{|c|c|c|c|c|c|c|c|c|}
\hline Nome Científico & $\begin{array}{c}\text { DA } \\
(\text { Ind. ha-1) }\end{array}$ & $\begin{array}{l}\text { DR } \\
(\%) \\
\end{array}$ & $\begin{array}{l}\text { FA } \\
(\%) \\
\end{array}$ & $\begin{array}{l}\text { FR } \\
(\%) \\
\end{array}$ & $\begin{array}{c}\text { DoA } \\
\left(\mathrm{m}^{2} \mathrm{ha}^{-1}\right)\end{array}$ & $\begin{array}{c}\text { DoR } \\
(\%) \\
\end{array}$ & $\begin{array}{l}\mathrm{VC} \\
(\%) \\
\end{array}$ & $\begin{array}{l}\mathrm{VI} \\
(\%) \\
\end{array}$ \\
\hline Croton blanchetianus & 1431 & 40,58 & 98 & 13,59 & 4,594 & 27,09 & 33,84 & 27,09 \\
\hline Mimosa ophthalmocentra & 730 & 20,7 & 99 & 13,73 & 1,662 & 9,8 & 15,25 & 14,74 \\
\hline Poincianella pyramidalis & 230 & 6,52 & 72 & 9,99 & 2,193 & 12,93 & 9,73 & 9,81 \\
\hline Allopbylus quercifolius & 327 & 9,27 & 51 & 7,07 & 1,508 & 8,89 & 9,08 & 8,41 \\
\hline Mimosa sp. 1 & 285 & 8,08 & 67 & 9,29 & 0,832 & 4,91 & 6,49 & 7,43 \\
\hline Aspidosperma pyrifolium & 59 & 1,67 & 31 & 4,3 & 1,707 & 10,07 & 5,87 & 5,35 \\
\hline Pilosocereus pachycladus & 38 & 1,08 & 31 & 4,3 & 1,349 & 7,95 & 4,51 & 4,44 \\
\hline Cereus jamacaru & 39 & 1,11 & 32 & 4,44 & 0,699 & 4,12 & 2,61 & 3,22 \\
\hline Lantana camara & 66 & 1,87 & 33 & 4,58 & 0,363 & 2,14 & 2,01 & 2,86 \\
\hline Manibot dichotoma & 52 & 1,47 & 34 & 4,72 & 0,147 & 0,86 & 1,17 & 2,35 \\
\hline Mimosa tenuiflora & 50 & 1,42 & 26 & 3,61 & 0,183 & 1,08 & 1,25 & 2,03 \\
\hline Acacia sp. & 31 & 0,88 & 22 & 3,05 & 0,276 & 1,62 & 1,25 & 1,85 \\
\hline Baubinia forficata & 47 & 1,33 & 17 & 2,36 & 0,309 & 1,82 & 1,58 & 1,84 \\
\hline Commiphora leptophloeos & 25 & 0,71 & 19 & 2,64 & 0,33 & 1,94 & 1,33 & 1,76 \\
\hline Capparis flexuosa & 29 & 0,82 & 21 & 2,91 & 0,159 & 0,94 & 0,88 & 1,56 \\
\hline Jatropha babiana & 22 & 0,62 & 18 & 2,5 & 0,022 & 0,13 & 0,38 & 1,08 \\
\hline Guapira sp. & 16 & 0,45 & 11 & 1,53 & 0,114 & 0,67 & 0,56 & 0,88 \\
\hline Capparis yco & 6 & 0,17 & 6 & 0,83 & 0,205 & 1,21 & 0,69 & 0,74 \\
\hline Eugenia sp. & 12 & 0,34 & 6 & 0,83 & 0,077 & 0,46 & 0,4 & 0,54 \\
\hline Coutarea bexandra & 10 & 0,28 & 7 & 0,97 & 0,03 & 0,17 & 0,23 & 0,48 \\
\hline Myracrodruon urundeuva & 5 & 0,14 & 5 & 0,69 & 0,069 & 0,4 & 0,27 & 0,41 \\
\hline Caesalpinea ferrea var. ferrea & 3 & 0,09 & 3 & 0,42 & 0,01 & 0,06 & 0,07 & 0,19 \\
\hline Mimosa sp. 3 & 1 & 0,03 & 1 & 0,14 & 0,066 & 0,39 & 0,21 & 0,19 \\
\hline Ziziphus joazeiro & 2 & 0,06 & 2 & 0,28 & 0,015 & 0,09 & 0,07 & 0,14 \\
\hline Anadenanthera colubrina & 2 & 0,06 & 2 & 0,28 & 0,012 & 0,07 & 0,06 & 0,13 \\
\hline Spondias tuberosa & 2 & 0,06 & 2 & 0,28 & 0,005 & 0,03 & 0,04 & 0,12 \\
\hline Helicteres eichleri & 1 & 0,03 & 1 & 0,14 & 0,02 & 0,12 & 0,07 & 0,1 \\
\hline Mimosa sp. 2 & 2 & 0,06 & 1 & 0,14 & 0,002 & 0,01 & 0,03 & 0,07 \\
\hline Croton rhamnifolioides & 1 & 0,03 & 1 & 0,14 & 0,002 & 0,01 & 0,02 & 0,06 \\
\hline Mimosa paraibana & 1 & 0,03 & 1 & 0,14 & 0,002 & 0,01 & 0,02 & 0,06 \\
\hline \multirow[t]{2}{*}{ Pseudobombax marginatum } & 1 & 0,03 & 1 & 0,14 & 0,001 & 0,01 & 0,02 & 0,06 \\
\hline & 3526 & 100 & 721 & 100 & 16,959 & 100 & 100 & 100 \\
\hline
\end{tabular}

$\mathrm{Na}$ área de Boa Vista foram amostrados majoritariamente espécies das famílias Fabaceae, Cactaceae e Anacardiaceae. As Cactaceae constituem a família de maior número, com destaque para T. palmadora e P. pachycladus. Observou-se uma relação entre o solo arenoso e o clima com o elevado número de cactáceas, evidenciadas no elevado teor de areia e menor precipitação pluvial ao longo de dez anos, sendo estas características relacionadas com o caráter xerófilo da vegetação (BARBOSA, 2011). A estrutura diamétrica e hipsométrica da área de Boa Vista demonstraram características sucessionais do fragmento, aparentando-se a forma de "J" reverso.

Segundo Assmann (1970), a distribuição da frequência dos indivíduos das comunidades arbóreas em curva de distribuição em forma exponencial negativa, parecendo um "J" invertido são típicas de formações florestais secundárias. O fragmento de caatinga de Boa Vista vem sendo sujeito ao longo dos anos ao corte eventual da madeira para a utilização em atividades agropecuárias, e neste sentido, apresentou baixa diversidade dentre as áreas. Entretanto, foram amostradas a maior diversidade de Cactaceae dentre as áreas em estudo, observou-se que os espécimes cresciam sobre um planossolo com presença de afloramentos rochosos. Em número de espécies por família, as Cactaceae, ocuparam o segundo lugar na área, ficando atrás apenas das Fabaceae. Deve-se destacar que as cactáceas representam algumas das únicas espécies remanescentes após ações antrópicas que causem transformações na vegetação original, sendo estas referidas como bioindicadoras do status da vegetação da Caatinga (ZAPPI, 2008).

A diversidade e a equabilidade são divergentes aos de outras áreas, em trabalhados realizados por Ferraz et al. (1998); Rodal et al. (1998); Alcoforado Filho et al. (2003), Pereira et al. (2000) e Cordeiro et al. (2017). No entanto, assemelham-se aos resultados encontrados por Silva et al. (2016) e Lacerda; Barbosa (2018) ao avaliarem a estrutura fitossociológica da vegetação em áreas de Caatinga. Convém elencar que as características climáticas, físico-químicas dos solos e a relação das comunidades rurais que estão no entorno dos fragmentos são preponderantes na diversidade florística dos mesmos (SANTOS et al., 2007).

Dentre as caatingas estudadas, Monteiro apresentou maior número de espécies, fato estritamente relacionado com os eventos antropogênicos pretéritos ao qual a área foi sujeita. Essa caatinga vem sendo mantida conservada, embora outrora houve corte seletivo de madeira para uso domiciliar e para pequenas construções. As Fabaceae e Euphorbiaceae são as famílias com maior número de espécies, entretanto foram amostradas espécies de comunidades estabelecidas como $S$. brasiliensis e C. glaziovii. Essa área apresentou indivíduos de maior porte em termos de diâmetro e altura, diversidade e equabilidade. Segundo 
Barbosa (2011) variáveis ambientais podem estar contribuindo para a riqueza florística de Monteiro, como precipitações médias anuais de $688,0 \mathrm{~mm}( \pm 149,1)$, e solos com alto teor em bases trocáveis e considerável aporte de material orgânico o que conferem a vegetação condicionantes para seu crescimento e desenvolvimento. De acordo com
Rodal et al. (1992) o maior ou menor número de espécies nos levantamentos realizados deve ser resposta a um conjunto de fatores, tais como situação topográfica, classe, profundidade e permeabilidade do solo e não apenas o total de chuvas, embora esse seja um dos fatores mais importantes nas regiões áridas e semiáridas.

Tabela 3. Espécies amostradas na área de vegetação natural do Sítio Caldeirão, Boa Vista - PB, com os respectivos parâmetros fitossociológicos em ordem decrescente de valor de importância.

Table 3. Species sampled in the area of natural vegetation of the Sítio Caldeirão, Boa Vista - PB, o with their phytosociological parameters in decreasing order of importance value.

\begin{tabular}{|c|c|c|c|c|c|c|c|c|}
\hline Nome Científico & $\begin{array}{c}\text { DA } \\
\left(\text { Ind. ha }{ }^{-1}\right)\end{array}$ & $\begin{array}{l}\text { DR } \\
(\%)\end{array}$ & $\begin{array}{l}\text { FA } \\
(\%)\end{array}$ & $\begin{array}{l}\text { FR } \\
(\%) \\
\end{array}$ & $\begin{array}{c}\text { DoA } \\
\left(\mathrm{m}^{2} \mathrm{ha}^{-1}\right)\end{array}$ & $\begin{array}{c}\text { DoR } \\
(\%)\end{array}$ & $\begin{array}{l}\mathrm{VC} \\
(\%) \\
\end{array}$ & $\begin{array}{c}\text { VI } \\
(\%) \\
\end{array}$ \\
\hline Tacinga palmadora & 1883 & 52,31 & 100 & 14,04 & 4,564 & 16,7 & 34,5 & 27,68 \\
\hline Poincianella pyramidalis & 502 & 13,94 & 97 & 13,62 & 8,943 & 32,72 & 23,33 & 20,1 \\
\hline Pilosocereus pachycladus & 171 & 4,75 & 79 & 11,1 & 3,819 & 13,97 & 9,36 & 9,94 \\
\hline Combretum duarteanum & 336 & 9,33 & 72 & 10,11 & 1,388 & 5,08 & 7,21 & 8,17 \\
\hline Mimosa ophthalmocentra & 207 & 5,75 & 80 & 11,24 & 1,625 & 5,95 & 5,85 & 7,64 \\
\hline Aspidosperma pyrifolium & 56 & 1,56 & 30 & 4,21 & 3,79 & 13,87 & 7,71 & 6,55 \\
\hline Jatropha mollissima & 102 & 2,83 & 51 & 7,16 & 0,157 & 0,57 & 1,7 & 3,52 \\
\hline Baubinia forficata & 84 & 2,33 & 30 & 4,21 & 0,164 & 0,6 & 1,47 & 2,38 \\
\hline Acacia sp. & 33 & 0,92 & 27 & 3,79 & 0,288 & 1,05 & 0,99 & 1,92 \\
\hline Manihot glaziovii & 41 & 1,14 & 25 & 3,51 & 0,116 & 0,42 & 0,78 & 1,69 \\
\hline Commiphora leptophloeos & 13 & 0,36 & 12 & 1,69 & 0,697 & 2,55 & 1,46 & 1,53 \\
\hline Guapira sp. & 23 & 0,64 & 18 & 2,53 & 0,202 & 0,74 & 0,69 & 1,3 \\
\hline Eugenia sp. & 14 & 0,39 & 9 & 1,26 & 0,562 & 2,06 & 1,22 & 1,24 \\
\hline Capsicum parvifolim & 19 & 0,53 & 17 & 2,39 & 0,044 & 0,16 & 0,34 & 1,03 \\
\hline Mimosa adenophyla & 18 & 0,5 & 12 & 1,69 & 0,19 & 0,69 & 0,6 & 0,96 \\
\hline Tacinga inamoena & 34 & 0,94 & 6 & 0,84 & 0,027 & 0,1 & 0,52 & 0,63 \\
\hline Pseudobombax marginatum & 7 & 0,19 & 6 & 0,84 & 0,119 & 0,43 & 0,31 & 0,49 \\
\hline Amburana cearensis & 7 & 0,19 & 7 & 0,98 & 0,021 & 0,08 & 0,14 & 0,42 \\
\hline Pilosocereus gounellei & 9 & 0,25 & 2 & 0,28 & 0,17 & 0,62 & 0,44 & 0,38 \\
\hline Myracrodruon urundeuva & 3 & 0,08 & 3 & 0,42 & 0,145 & 0,53 & 0,31 & 0,35 \\
\hline Allophylus quercifolius & 7 & 0,19 & 4 & 0,56 & 0,053 & 0,19 & 0,19 & 0,32 \\
\hline Schinopsis brasiliensis & 5 & 0,14 & 5 & 0,7 & 0,024 & 0,09 & 0,11 & 0,31 \\
\hline Anadenanthera colubrina & 4 & 0,11 & 3 & 0,42 & 0,086 & 0,32 & 0,21 & 0,28 \\
\hline Mimosa tenuiflora & 5 & 0,14 & 3 & 0,42 & 0,016 & 0,06 & 0,1 & 0,21 \\
\hline Sapium lanceolatum & 4 & 0,11 & 3 & 0,42 & 0,026 & 0,09 & 0,1 & 0,21 \\
\hline Maytenus rigida & 3 & 0,08 & 3 & 0,42 & 0,008 & 0,03 & 0,06 & 0,18 \\
\hline Helicteres eichleri & 2 & 0,06 & 2 & 0,28 & 0,003 & 0,01 & 0,03 & 0,12 \\
\hline Spondias tuberosa & 1 & 0,03 & 1 & 0,14 & 0,051 & 0,19 & 0,11 & 0,12 \\
\hline Caesalpinia ferrea var. ferrea & 2 & 0,06 & 2 & 0,28 & 0,002 & 0,01 & 0,03 & 0,11 \\
\hline Melocactus zehntneri & 1 & 0,03 & 1 & 0,14 & 0,017 & 0,06 & 0,04 & 0,08 \\
\hline Lantana camara & 3 & 0,08 & 1 & 0,14 & 0,004 & 0,02 & 0,05 & 0,08 \\
\hline \multirow[t]{2}{*}{ Cereus jamacaru } & 1 & 0,03 & 1 & 0,14 & 0,009 & 0,03 & 0,03 & 0,07 \\
\hline & 3600 & 100 & 712 & 100 & 27,33 & 100 & 100 & 100 \\
\hline
\end{tabular}

Santos et al. (2017) ao estudarem a composição florística de um fragmento de Caatinga no município de Upanema$\mathrm{RN}$, verificaram que a família com maior número de espécies e de indivíduos foi a Fabaceae e as espécies com maior importância na área de estudo sob os aspectos fitossociológicos foram C. leprosum, A. pyrifolium, B. cheilantha, M. ophthalmocentra e $P$. pyramidalis. Segundo esses autores, a diversidade de espécie encontrada na área foi considerada média se comparada com outros fragmentos florestais de caatinga já estudados.

As áreas de caatinga estudadas apresentaram algum grau de intervenção antrópica ao longo dos anos. A área do INSA/MCT foi recentemente utilizada para fins agrícolas, onde provavelmente a ocorrência de espécies das famílias Fabaceae, Euphorbiacae e Anacardiaceae denotaram o caráter sucessional da vegetação. Os resultados desta pesquisa corroboram com outros estudos de florística na caatinga, apontando para as Fabaceae, Euphorbiaceae, Cactaceae e Malvaceae como de grande representatividade do bioma (RODAL et al., 1992; ARAÚJO et al., 1995; FERRAZ et al., 1998; ALCOFORADO FILHO et al., 2003;
GIULIETTI, 2006; SANTANA; SOUTO, 2006; FABRICANTE; ANDRADE, 2007).

\section{CONCLUSÕES}

As famílias mais representativas das áreas avaliadas são Euphorbiaceae, Fabaceae e Cactaceae, sendo as espécies representativas Croton blanchetianus, $P$. pyramidalis, $M$. ophthalmocentra, T. palmadora e P. pachycladus.

A caatinga de Boa Vista apresenta riqueza e diversidade de Cactaceae. A caatinga de Monteiro apresenta diversidade florística e melhor estado conservação do patrimônio genético autóctone.

As espécies C. blanchetianus, C. rbamnifolioides, $A$. quercifolius, Mimosa sp., C. duarteanum, B. forficata, B. cheilantha, L. camara, R. laurifolia, T. inamoena e $P$. gounellei apresentam padrão de distribuição agregada nas áreas de caatinga de Campina Grande, Boa Vista e Monteiro - PB.

As áreas são similares floristicamente, entretanto a vegetação da área de Campina Grande e Boa Vista são encontradas espécimes de ambientes em regeneração. 
Tabela 4. Espécies amostradas na área de vegetação natural do Sítio Mocó de Baixo, Monteiro - PB, com os respectivos parâmetros fitossociológicos em ordem decrescente de valor de importância.

Table 4. Species sampled in the area of natural vegetation of the Sítio Mocó de Baixo, Monteiro - PB, with their phytosociological parameters in decreasing order of importance value.

\begin{tabular}{|c|c|c|c|c|c|c|c|c|}
\hline Nome Científico & $\begin{array}{c}\text { DA } \\
\text { (Ind. ha-1) }\end{array}$ & $\begin{array}{l}\text { DR } \\
(\%) \\
\end{array}$ & $\begin{array}{l}\text { FA } \\
(\%) \\
\end{array}$ & $\begin{array}{l}\text { FR } \\
(\%) \\
\end{array}$ & $\begin{array}{c}\text { DoA } \\
\left(\mathrm{m}^{2} \mathrm{ha}^{-1}\right)\end{array}$ & $\begin{array}{c}\text { DoR } \\
(\%) \\
\end{array}$ & $\begin{array}{l}\mathrm{VC} \\
(\%) \\
\end{array}$ & $\begin{array}{c}\mathrm{VI} \\
(\%) \\
\end{array}$ \\
\hline Croton rhamnifolioides & 1086 & 31,02 & 83 & 9,06 & 6,497 & 22,59 & 26,81 & 20,89 \\
\hline Poincianella pyramidalis & 287 & 8,2 & 89 & 9,72 & 8,402 & 29,22 & 18,71 & 15,71 \\
\hline Croton blanchetianus & 637 & 18,19 & 82 & 8,95 & 2,393 & 8,32 & 13,26 & 11,82 \\
\hline Mimosa ophthalmocentra & 287 & 8,2 & 107 & 11,68 & 3,264 & 11,35 & 9,78 & 10,41 \\
\hline Baubinia cheilantha & 395 & 11,28 & 78 & 8,52 & 1,282 & 4,46 & 7,87 & 8,09 \\
\hline Manihot dichotoma & 197 & 5,63 & 79 & 8,62 & 1,724 & 6 & 5,81 & 6,75 \\
\hline Malpighiaceae (sp. 1) & 183 & 5,23 & 68 & 7,42 & 0,346 & 1,2 & 3,22 & 4,62 \\
\hline Parapiptadenia zebntneri & 37 & 1,06 & 30 & 3,28 & 0,829 & 2,88 & 1,97 & 2,41 \\
\hline Cereus jamacaru & 38 & 1,09 & 29 & 3,17 & 0,475 & 1,65 & 1,37 & 1,97 \\
\hline Jatropha mollissima & 49 & 1,4 & 33 & 3,6 & 0,144 & 0,5 & 0,95 & 1,83 \\
\hline Spondias tuberosa & 24 & 0,69 & 22 & 2,4 & 0,685 & 2,38 & 1,53 & 1,82 \\
\hline Helietta apiculata & 34 & 0,97 & 21 & 2,29 & 0,55 & 1,91 & 1,44 & 1,73 \\
\hline Myracrodruon urundeuva & 31 & 0,89 & 26 & 2,84 & 0,293 & 1,02 & 0,95 & 1,58 \\
\hline Anadenanthera colubrina var. cebil & 24 & 0,69 & 18 & 1,97 & 0,545 & 1,9 & 1,29 & 1,52 \\
\hline Guapira sp. & 33 & 0,94 & 26 & 2,84 & 0,19 & 0,66 & 0,8 & 1,48 \\
\hline Capparis cynophallophora & 24 & 0,69 & 19 & 2,07 & 0,26 & 0,9 & 0,79 & 1,22 \\
\hline Mimosa tenuiflora & 18 & 0,51 & 16 & 1,75 & 0,295 & 1,03 & 0,77 & 1,1 \\
\hline Helicteres ovata & 27 & 0,77 & 13 & 1,42 & 0,026 & 0,09 & 0,43 & 0,76 \\
\hline Chloroleucon foliolosum & 11 & 0,31 & 11 & 1,2 & 0,035 & 0,12 & 0,22 & 0,55 \\
\hline Commiphora leptophloeos & 9 & 0,26 & 9 & 0,98 & 0,079 & 0,28 & 0,27 & 0,51 \\
\hline Schinopsis brasiliensis & 8 & 0,23 & 8 & 0,87 & 0,109 & 0,38 & 0,3 & 0,49 \\
\hline Luetzelburgia auriculata & 14 & 0,4 & 8 & 0,87 & 0,012 & 0,04 & 0,22 & 0,44 \\
\hline Rollinia laurifolia & 11 & 0,31 & 5 & 0,55 & 0,039 & 0,14 & 0,23 & 0,33 \\
\hline Aspidosperma pyrifolium & 4 & 0,11 & 4 & 0,44 & 0,101 & 0,35 & 0,23 & 0,3 \\
\hline Allopbylus quercifolius & 6 & 0,17 & 5 & 0,55 & 0,022 & 0,08 & 0,12 & 0,27 \\
\hline Harrisia adscendens & 5 & 0,14 & 5 & 0,55 & 0,008 & 0,03 & 0,08 & 0,24 \\
\hline Rollinia sp. & 4 & 0,11 & 4 & 0,44 & 0,018 & 0,06 & 0,09 & 0,2 \\
\hline Senna macranthera & 4 & 0,11 & 4 & 0,44 & 0,005 & 0,02 & 0,07 & 0,19 \\
\hline Torresea cearensis & 2 & 0,06 & 2 & 0,22 & 0,046 & 0,16 & 0,11 & 0,15 \\
\hline Ziziphus joazeiro & 3 & 0,09 & 3 & 0,33 & 0,007 & 0,02 & 0,05 & 0,15 \\
\hline Cordia leucocephala & 2 & 0,06 & 2 & 0,22 & 0,004 & 0,01 & 0,03 & 0,1 \\
\hline Ceib a glaziovii & 1 & 0,03 & 1 & 0,11 & 0,048 & 0,17 & 0,1 & 0,1 \\
\hline sp. 4 & 2 & 0,06 & 2 & 0,22 & 0,002 & 0,01 & 0,03 & 0,09 \\
\hline Pilosocereus piaubyensis & 1 & 0,03 & 1 & 0,11 & 0,01 & 0,04 & 0,03 & 0,06 \\
\hline sp. 3 & 1 & 0,03 & 1 & 0,11 & 0,005 & 0,02 & 0,02 & 0,05 \\
\hline sp. 5 & 1 & 0,03 & 1 & 0,11 & 0,003 & 0,01 & 0,02 & 0,05 \\
\hline \multirow[t]{2}{*}{ Sapium lanceolatum } & 1 & 0,03 & 1 & 0,11 & 0,004 & 0,01 & 0,02 & 0,05 \\
\hline & 3501 & 100 & 916 & 100 & 28,756 & 100 & 100 & 100 \\
\hline
\end{tabular}

\section{REFERÊNCIAS}

ALCOFORADO FILHO, F. G.; SAMPAIO, E. V. S. B.; RODAL, M. J. N. Florística e fitossociologia de um remanescente de vegetação caducifólia arbórea em Caruaru, Pernambuco. Acta Botânica Brasílica, São Paulo, v. 17, n. 2, p. 287-303, 2003. DOI: https://doi.org/10.1590/S0102-33062003000200011

ARAÚJO, E. L.; SAMPAIO, E. V. S. B.; RODAL, M. J. N. Composição florística e fitossociológica de três áreas de Caatinga de Pernambuco. Revista Brasileira de Biologia, São Carlos, v. 55, n. 4, p. 595-607, 1995.

ARAUJO, K. D.; PARENTE, H. N.; ÉDER-SILVA, É.; RAMALHO, C. I.; DANTAS, R. T.; ANDRADE, A. P. de; SILVA, D. S. da. Levantamento florístico do estrato arbustivo-arbóreo em áreas contíguas de Caatinga no Cariri Paraibano. Revista Caatinga, Mossoró, v. 23, n. 1, p. 63-70, 2010.

ASSMANN, E. The principles of forest yield: studies in the organic production, structure, increment and yield of forest stands. Braunschweig: Pergamon Press, 1970. 506 p.
BARBOSA, A. S. Estrutura da vegetação e distribuição espacial de Cactaceae em áreas de caatinga do semiárido paraibano. 2011. 166f. (Dissertação de Mestrado em Agronomia) - Programa de Pós-Graduação em Agronomia. Centro de Ciências Agrárias Universidade Federal da Paraíba, Areia, 2011.

CIENTEC. Sistema para análise fitossociológica $\mathbf{e}$ elaboração dos planos de manejo de florestas nativas. Viçosa, MG, 2006. $131 \mathrm{p}$.

CORDEIRO, J. M. P.; SOUZA, B. I.; FELIX, L. P. Florística e fitossociologia em floresta estacional decidual na Paraíba, nordeste do Brasil. Gaia Scientia, v. 11, n. 1, p. 1-16, 2017.

CPRM - Serviço Geológico do Brasil Projeto cadastro de fontes de abastecimento por água subterrânea. Diagnóstico do município de Campina Grande, estado da Paraíba. João de Castro Mascarenhas, Breno Augusto Beltrão, Luiz Carlos de Souza Junior, Franklin de Morais, Vanildo Almeida Mendes, Jorge Luiz Fortunato de Miranda (Orgs.) Recife: CPRM/PRODEEM, 2005a. 20 p. 
CPRM - Serviço Geológico do Brasil Projeto cadastro de fontes de abastecimento por água subterrânea. Diagnóstico do município de Monteiro, estado da Paraíba. João de Castro Mascarenhas, Breno Augusto Beltrão, Luiz Carlos de Souza Junior, Franklin de Morais, Vanildo Almeida Mendes, Jorge Luiz Fortunato de Miranda (Orgs.) Recife: CPRM/PRODEEM, 2005b. 11 p.

FABRICANTE, J. R.; ANDRADE, L. A. Análise estrutural de um remanescente de caatinga no Seridó paraibano. Oecologia Brasiliensis, v. 11, n. 3, p. 341-349, 2007.

FERRAZ, E. M. N.; RODAL, M. J. N.; SAMPAIO, E. V. S. B; PEREIRA, R. C. A. Composição florística em trechos de vegetação de caatinga e brejo de altitude na região do Vale do Pajeú, Pernambuco. Revista Brasileira de Botânica, São Paulo, v. 21, n. 1, p. 7-15, 1998.

GARIGLIO, M. A.; SAMPAIO, E. V. S. B.; CESTARO, L. A.; KAGEYAMA, P. Y. Uso sustentável e conservação dos recursos florestais da caatinga. Brasília: Serviço Florestal Brasileiro, 2010.368 p.

GIULIETTI, A. M.; CONCEIÇÃO, A.; QUEIROZ, L. P. Diversidade e caracterização das fanerógamas do semi-árido brasileiro. Recife: Associação Plantas do Nordeste, 2006. 488 p.

GOVERNO DO ESTADO DA PARAÍBA. Plano Estadual de Recursos Hídricos. Governo da Paraíba/Secretaria de Estado da Ciência e Tecnologia do Meio Ambiente/Secretaria Executiva da Gestão das Águas do Estado da Paraíba. Brasília: Consórcio TC/BR, 2006, 112 p.

LACERDA, A. V; BARBOSA, F. M. Fitossociologia da vegetação arbustivo-arbórea em uma área de mata ciliar no semiárido paraibano, Brasil. Gaia Scientia, v. 12, n. 2, p. 34-43, 2018.

MAGURRAN, A. Ecological diversity and its measurement. New Jersey: Princeton University Press, 1988. 179 p.

MARANGON, L. C.; SOARES, J. J.; FELICIANO, A. L. P. F. Florística arbórea da mata da pedreira, município de Viçosa, Minas Gerais. Revista Árvore, Viçosa, v. 27, n. 2, p. 207-215, 2003. DOI: https://dx.doi.org/10.1590/S0100-67622003000200010

MISSOURI BOTANICAL GARDEN. Base de dados Tropicos. Disponível em: <http://www.tropicos.org/> Acessado em 21 de junho de 2014.

MUlLER-DOMBOIS, D.; ELLENBERG, H. Aims and methods of vegetation ecology. New York: Wiley, 1974. 574 p.

PEREIRA, I. M. Levantamento florístico do estrato arbustivo-arbóreo e análise da estrutura fitossociológica de ecossistema de caatinga sob diferentes níveis de antropismo. 2000. 70f. Dissertação (Mestrado em Agronomia). Universidade Federal da Paraíba, 2000.

PESSOA, M. F.; GUERRA, A. M. N. M.; MARACAJÁ, P. B.; LIRA, J. F; B.; DINIZ FILHO, E. T. Estudo da cobertura vegetal em ambientes da caatinga com diferentes formas de manejo no assentamento Moacir Lucena, Apodi - RN. Revista Caatinga, Mossoró, v. 21, n. 3, p. 40-48, 2008.
PIELOU, E. C. Ecological diversity. New York: Wiley, 1975. $165 \mathrm{p}$.

RODAL, M. J. N.; ANDRADE, K. V. A.; SALES, M. F.; GOMES, A. P. S. Fitossociologia do componente lenhoso de um refúgio vegetacional no município de Buíque, PE. Revista Brasileira Biologia, v. 58, n. 3, p. 517-526, 1998.

RODAL, M. J. N.; SAMPAIO, E. V. S.; FIGUEIREDO, M. A. Manual sobre métodos de estudo florístico e fitossociológico: ecossistema caatinga. Brasília: Sociedade Botânica do Brasil, 1992. 24 p.

SANTANA, J. A. S.; SOUTO, J. S. Diversidade e estrutura fitossociológica da caatinga na Estação Ecológica do Seridó-RN. Revista de Biologia e Ciências da Terra, v. 6, n. 2, p. 232-242, 2006.

SANTOS, R. M.; VIEIRA, F. A.; FAGUNDES, A.; NUNES, Y. R. F.; GUSMÃO, E. Riqueza e similaridade florística de oito remanescentes florestais no norte de minas gerais, Brasil. Revista Árvore, Viçosa-MG, v. 31, n. 1 p. 135-144, 2007. DOI: https://doi.org/10.1590/S0100-67622007000100015

SANTOS, W. S.; SOUZA, M. P.; NÓBREGA, G. F. Q.; MEDEIROS, F. S.; ALVES, A. R.; HOLANDA, A. C. Caracterização florístico-fitossociológica do componente lenhoso em fragmento de caatinga no município de Upanema-RN. Nativa, Sinop, v. 5, n. 2, p. 85-91, 2017. DOI: http://dx.doi.org/10.5935/2318-7670.v05n02a02

SILVA, A. C. C.; PRATA, A. P.; MELLO, A. A. Florística, fitossociologia e caracterização sucessional em um remanescente de Caatinga em Sergipe. Gaia Scientia, v. 10, n. 4, p. 1-14, 2016.

SILVA, L. S.; ALVES, A. R.; NUNES, A. K. A.; MACEDO, W. S.; MARTINS, A. R. Florística, estrutura e sucessão ecológica de um remanescente de mata ciliar na bacia do rio Gurguéia-PI. Nativa, Sinop, v. 3, n. 3, p. 156-164, 2015. DOI: http://dx.doi.org/10.14583/23187670.v03n03a02

SILVA, G. M. C.; MARTINS, P. L.; SILVA, H.; FREITAS, K. K. C. Estudo autoecológico de Bumélia sertorium (Quixabeira) - Espécie ameaçada de extinção no ecossistema Caatinga. Biologia e Ciências da Terra, Paraíba, v. 4, n. 1, p. 0, 2004.

SOUZA, M. T. C.; SILVA, A. M.; LIMA JÚNIOR, D. M.; ANDRADE, A. P. Variability of a vegetation in Caatinga areas in the Northeast of Brazil. Revista Agrária Acadêmica, v. 2, n. 3, p. 115-121, 2019. DOI: https://doi.org/10.32406/v2n32019/115$121 /$ agrariacad

THE ANGIOSPERM PHYLOGENY GROUP_CHASE, M. W.; CHRISTENHUSZ, M. J. M.; FAY M. F.; BYNG J. W.; JUDD, W. S.; SOLTIS, D. E.; MABBERLEY, D. J.; SENNIKOV, A. N.; SOLTIS, P. S.; STEVENS, P. F. An update of the Angiosperm Phylogeny Group classification for the orders and families of flowering plants: APG IV, Botanical Journal of the Linnean Society, London, v. 181, n. 1, p. 1-20, 2016. DOI: https://dx.doi.org/10.1111/boj.12385

ZAPPI, D. Fitofisionomia da Caatinga a Cadeia do Espinhaço. Megadiversidade, v. 4, n. 1-2, p. 34-38, 2008. 